\title{
The BER Analysis of MIMO System for M-PSK over Different Fading Channels using STBC Code Structure
}

\author{
Vijaya Durga Ravva, Allan McLauchlin
}

\begin{abstract}
High information rates inside the restricted frequency (RF) spectrum is often fascinating that results in radios with capabilities on the far side a single-input single-output (SISO) topology. In recent days introduced wireless systems have adopted multiple-input multiple-output (MIMO) topologies that use 2 or more transmitters and 2 or more receivers to send information at the same time over same RF bandwidth. The performance of MIMO system may be improved by involving multiple antennas at transmitter and receiver therefore on offer spatial diversity. during this paper, the performance analysis of MIMO system over AWGN attenuation channel and Rician Channel with $\mathrm{ZF}$ receiver is bestowed. The consequences of the antenna choice can even be analyzed from the simulated results. The BER (Bit Error Rate) performance characteristics of ZeroForcing (ZF) receiver is investigated for M-PSK modulation technique over the AWGN channel and Rician Channel.
\end{abstract}

Keywords - MIMO, AWGN, Rician Channel, spatial diversity, $B E R, Z F, M-P S K$, SNR, multi-path attenuation, STBC.

\section{INTRODUCTION}

Multiple antennas used at transmitter and receiver sides square measure utilized in wireless communication therefore on succeed the high information rates through spatial multiplexing [1]. MIMO involves multiple transmitters causation distinctive information content to multiple receivers using spatial multiplexing. MIMO will increase information rates and needs higher SNR than constant SISO transmission. This technique offers higher capability to wireless systems and therefore the capability will increase linearly with the amount of antennas.

Future trends of wireless communications mentioned below results in MIMO development.-

- Future wireless applications produce insatiability as demand for "high information rate" and "high link qualiSTBty" wireless access has increased plenty.

- Spectrum has become a scarce and pricey resource as information measure is incredibly restricted

Manuscript published on 30 September 2019

* Correspondence Author

Vijaya Durga Ravva*, Department of Electronics and Communication Engineering, University of Hertfordshire, College Ln, Hatfield AL10 9AB, UK.

Allan McLauchlin, Department of Electronics and Communication Engineering, University of Hertfordshire, College Ln, Hatfield AL10 9AB, UK.

(C) The Authors. Published by Blue Eyes Intelligence Engineering and Sciences Publication (BEIESP). This is an open access article under the CC-BY-NC-ND license http://creativecommons.org/licenses/by-nc-nd/4.0/
Regulation, device and system capability considerations as transmit power is proscribed

- Time and frequency domain process square measure at limits, however area is not!

A MIMO system utilizes spatial diversity by using spatially separated antennas during a dense multipath scattering surroundings [2]. In MIMO, phased sets of

antennas profit of the variations within the spatial propagation methods to boost signal hardiness or to send multiple information sets over single frequency band.In general, having multiple antennas offers 3 useful cases: diversity, beam forming, and space division multiplexing (SDM). MIMO systems square measure enforced to get a diversity gain to combat signal attenuation.

The efficient Implementation of Space time Block coding(STBC) for broadband wireless communications improves the performance and diversity gains of a space time (ST) coding system through variety of parameters together with variety of trellis codes and channel fading[3][4]. The 2 main functions of STC: diversity \& multiplexing. the most performance wants tradeoffs between diversity and multiplexing. V-BLAST could be a system within which full spatial diversity is typically not achieved [5].

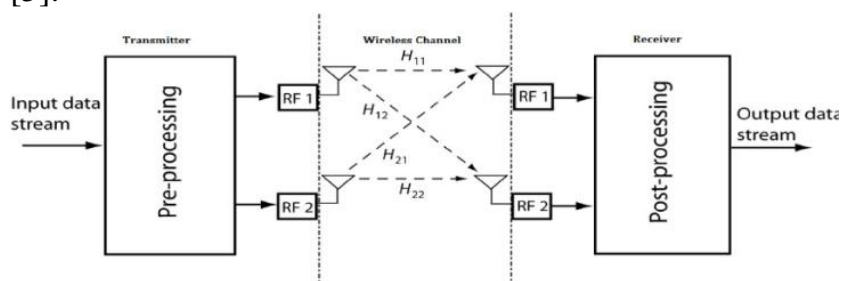

Fig. 1.1: 2X2 MIMO System

The diagram of MIMO system is shown in Fig. 1.1. the fundamental main plan of MIMO is to boost BER or rate (bits/sec) by using multiple TX/RX antennas. [6]. For achieving high rate or improved BER, the core theme utilized in MIMO system is Space Time Coding (STC).

MIMO provides higher capability (bits/s/Hz), higher transmission quality (BER), increased coverage and improved user position estimation due to the

- Spatial multiplexing gain: capacity gain at no further power or information measure consumption obtained through the use of multiple antennas at each side of a wireless link

- Diversity gain : Betterment in link reliability obtained by transmission identical information on severally attenuation branches 


\section{The BER Analysis of MIMO System for M-PSK over Different Fading Channels using STBC Code}

Structure

\section{- Array gain}

- Interference reduction

In this paper, the effects of AWGN channel are considered on the performance of MIMO systems with totally different antenna selection using ZF receivers. AWGN channel could be a universal channel that adds a white Gaussian noise to the signal passing through it. It's a channel model used for analyzing the assorted modulation

In this paper, the effects of AWGN channel are considered on the performance of MIMO systems with totally different antenna selection using ZF receivers. AWGN channel could be a universal channel that adds a white Gaussian noise to the signal passing through it. It's a channel model used for analyzing the assorted modulation schemes.

\section{LITERATURE REVIEW}

Multiple input-multiple output (MIMO) communication systems using coding techniques

applicable to multiple antenna transmissions have recently been embraced as an efficient means that to realize high rate over wireless channels.

A. I. Sulyman [7] describes the impact of antenna choice on the performance of multiple input-multiple output (MIMO) systems over nonlinear communication channels. The author has derived precise analytical expressions for evaluating the PWEP performance of reference frame trellis codes over nonlinear MIMO channel.

C. Wang [8] explained the approach that extend the capability of MIMO systems by using the spatial multiplexing where individual information streams are transmitted from the antennas. These information streams will be then separated at the receiver by appropriate signal process techniques like most likelihood (ML) which achieves optimum performance or linear receivers like ZeroForcing (ZF) which offer sub-optimal performance however it additionally offers vital machine complexness reduction with tolerable performance degradation.

The comparison of MIMO with SISO technology was mentioned by S. G. Kim et. al [9]. MIMO can't solely improve spectral potency, however additionally enhance link output or capability of the system. The authors bestowed a decent closed kind BER approximation of MPSK for MIMO ZF receiver over continuous flat attenuation channels. The larger the distinction between the numbers of transmit antennas and the number of receiver is, the higher performance is.

A simple two-branch transmit diversity technique was bestowed by S. Alamouti [10]. The technique uses 2 transmit antennas and one receive antenna. It provides identical diversity order as maximal-ratio receiver combining (MRRC) with one transmit antenna, and 2 receive antennas.

transmitter and zero-forcing process at the receiver in multiuser MIMO programming systems was mentioned by C. Chen [12].

V. Tarokh et.al [11] design a channel codes for betterment of the high rate and therefore the reliableness of communications over attenuationchannels using multiple transmit antennas. information is encoded by a channel code and therefore the encoded information is split into multiple streams that square measure at the same time transmitted using multiple transmit antennas. The received signal at every receive antenna could be a linear superposition of the multiple transmittedsignals rattled by noise.

The performance analysis of the inexpensive and effective transmission strategy that employs the The performance analysis of the inexpensive and effective transmission strategy that employs the straightforward spatial multiplexing at the transmitter and zero-forcing process at the receiver in multiuser MIMO programming systems was mentioned by C. Chen [12].

N. S. Kumar et. al [13], investigated regarding the 3 types of equalizer for MIMO wireless receivers. The authors clearly mentioned a couple of fastened antenna MIMO antenna configuration and compared the performance with all the 3 types of equalizer primarily based receiver specifically ZF, ML, and MMSE. BER performance of milliliter Equalizer is superior than zero forcing Equalizer and Minimum Mean square Equalizers. Based on the mathematical modeling and therefore the simulation result it's inferred that the milliliter equalizer is that the better of the 3 equalizers.

\section{MODULATION TECHNIQUE}

The digital electronic communication system consists of 2 fundamentals elements i.e. modulation and encoder. Modulation is that the method of mapping the digital information to analog kind therefore it may be transmitted over an extended distance via channel. Modulator could be a device utilized in transmitter facet to perform modulation and reception is performed at the receiver end by creating use of demodulator . reception is that the inverse method of modulation therefore to recover the transmitted digital information.

Phase-shift keying (M-PSK) that the signal set is:

$$
\mathrm{x}_{i}(t)=\frac{\sqrt{2 E_{s}}}{\mathrm{~T}_{S}} \cos \left(2 \pi * \mathrm{f}_{\mathrm{c \tau}}+\frac{2(\mathrm{i}-1)}{\mathrm{M}}\right)
$$

$\mathrm{i}=1,2, \ldots . \mathrm{M} \& 0<\mathrm{t}<T_{S}$

where $E_{s}$ the signal energy per symbol $T_{s}$ is the symbol duration and $\mathrm{f}_{\mathrm{c} \tau}$ is the carrier frequency.

This phase of the carrier takes on one of the $\mathrm{M}$ possible values.

$$
\theta=2(\mathrm{i}-1)^{\pi / \mathrm{M}} \quad i=1,2, \ldots M
$$

M-ary PSK modulation yields a circular constellation as shown in Fig. 1.2. the most constraint was to maintained the amplitude of the transmitted signals be constant.

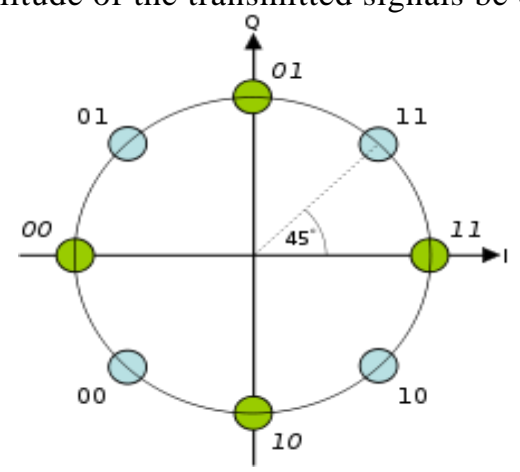

Fig. 1.2: Signal Constellation Diagram for 4-PSK 

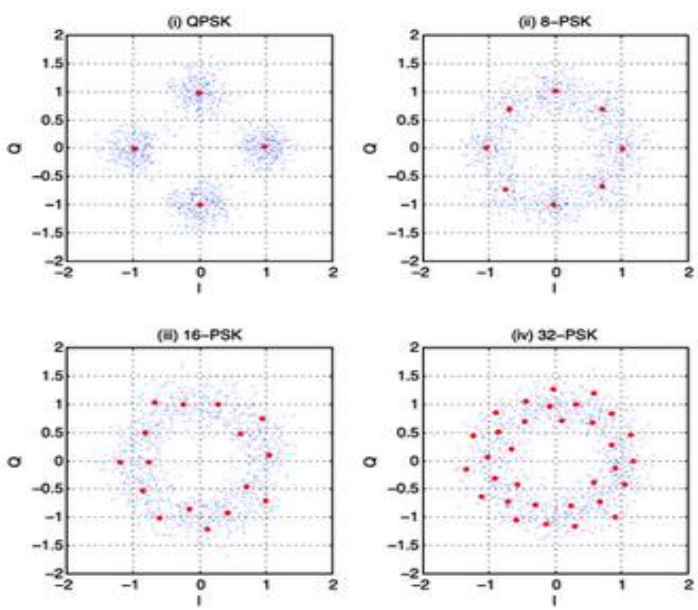

Fig. 1.3: Signal Constellation Diagram for M-PSK

IV.

AWGN CHANNEL

Channel could be a channel that adds a white Gaussian noise to the signal passing through it. this means that the channel's amplitude frequency response is flat (thus with unlimited or infinite bandwidth) and phase frequency response is linear for all frequencies so modulated signals have it with none amplitude loss and phase distortion of frequency elements.

The fading is avoided. the sole distortion is introduced by the AWGN.The received signal is simplified to

$$
r(t)=x(t)+n(t)
$$

where $n(t)$ is that the additive white Gaussian noise.

The whiteness of $\mathrm{n}(\mathrm{t})$ implies that it's a stationary random method with a flat power spectral density (PSD) for all frequencies. it's a convention to assume its PSD as $\mathrm{N}(\mathrm{t})=\mathrm{N} 0 / 2$, $-\infty<\mathrm{f}<\infty$

This implies that a white method has infinite power. This in fact could be a mathematical idealization. in line with the Wiener-Khinchine theorem, the autocorrelation function of the AWGN is

$$
\mathrm{R}(\tau)=\mathrm{N} 0 / 2 \delta(\tau)
$$

where $\delta(\tau)$ is the Dirac delta function.

\section{MIMO SYSTEM MODEL}

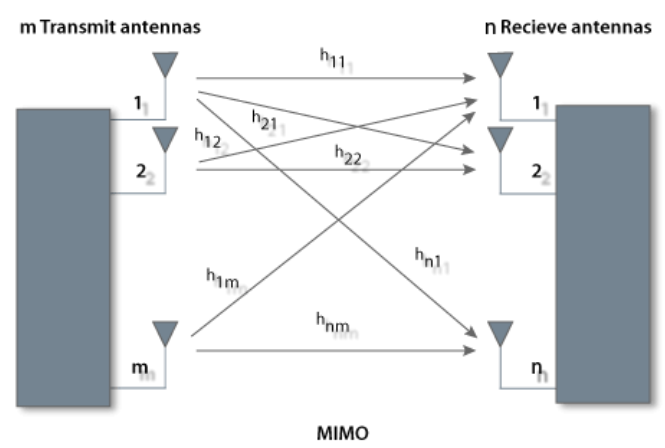

Fig.1. MIMO system

The 2X2 MIMO channel is delineated in Fig. 1.3 with an array with a pair of components at the transmitter and an antenna array with a pair of components at the receiver is taken into account. The input-output notation of the MIMO system will currently be expressed by the equation

$$
\mathrm{y}(\mathrm{t})=\mathrm{H}(\tau, \mathrm{t}) * \mathrm{~s}(\mathrm{t})+\mathrm{u}(\mathrm{t})
$$

where $*$ denotes convolution, $\mathrm{s}(\mathrm{t})$ is a $\mathrm{n}_{\mathrm{t}} \mathrm{X} 1$ vector with respect to the $n_{t}$ transmitted signals, $y(t)$ is a $n_{r} X 1$ vector vector with respect to the $\mathrm{n}_{\mathrm{r}}$ and $\mathrm{u}(\mathrm{t})$ is the additive white noise.

The impulse response of the channel between the $\mathrm{j}^{\text {th }}$ transmitter element and the $i^{t h}$ receiver element is denoted as $h_{i j}(\tau, t)$. The MIMO channel can then be described by the $n_{r} X n_{t} H(\tau, t)$ matrix :

$$
H(\tau, t)=\left[\begin{array}{ccc}
h_{11}(\tau, t) & h_{12}(\tau, t) \ldots \ldots & h_{1 n_{t}}(\tau, t) \\
h_{21}(\tau, t) & h_{22}(\tau, t) & h_{2 n_{t}}(\tau, t) \\
h_{n_{r} n_{1}}(\tau, t) & h_{n_{r} n_{2}}(\tau, t) & h_{n_{r} n_{t}}(\tau, t)
\end{array}\right]
$$

The matrix elements are complex numbers that correspond to the attenuation and phase shift that the wireless channel introduces to the signal reaching the receiver with delay $\tau$.

\section{ZERO FORCING EQUALIZER}

Zero Forcing Equalizer could be a linear equalization algorithmic rule that inverts the frequency response of the channel utilized in communication systems.

The Zero-Forcing Equalizer applies the inverse of the channel to the received signal, to revive the signal before the channel. The name Zero Forcing corresponds to conveyance down the ISI to zero during a noise free case. this can be helpful once ISI is important compared to noise [8] [9].

For a channel with frequency response $F(f)$ the zero forcing equalizer $C(f)$ is built specified $C(f)=1 / F(f)$. so the combination of channel and equalizer offers a flat frequency response and linear phase $\mathrm{F}(\mathrm{f}) \mathrm{C}(\mathrm{f})=1$. By using the linear model the received vector may be delineated as:

$\mathrm{Y}=\mathrm{Hx}+\mathrm{n}$

For a 2x2 MIMO channel, the channel is modeled as,

$$
\left(\begin{array}{l}
y_{1} \\
y_{2}
\end{array}\right)=\left(\begin{array}{ll}
h_{1,1} & h_{1,2} \\
h_{2,1} & h_{2,2}
\end{array}\right)\left(\begin{array}{l}
X_{1} \\
X_{2}
\end{array}\right)+\left(\begin{array}{l}
n_{1} \\
n_{2}
\end{array}\right)
$$

\section{SIMULATED RESULTS}

In this section, BER (Bit Error Rate) analysis of MIMO system over AWGN channel and Rician channel using STBC code structure is performed for M-PSK Modulation techniques. The BER analysis of MIMO system is performed for M-PSK over AWGN fading channel wherever M may be 32, 64, 128, 256, 512 and 1024 for various antenna configurations. Here receiving antennas used ranging from $\mathrm{NR}=1$ to $\mathrm{NR}=4$. The BER analysis of MIMO system is performed for M-

PSK over AWGN fading channel wherever M may be 32, 64, 128, 256, 512 and 1024 for various antenna configurations. Here receiving antennas used ranging from $\mathrm{NR}=1$ to $\mathrm{NR}=4$ 
The BER Analysis of MIMO System for M-PSK over Different Fading Channels using STBC Code Structure

7.2 M-PSK OVER AWGN CHANNEL

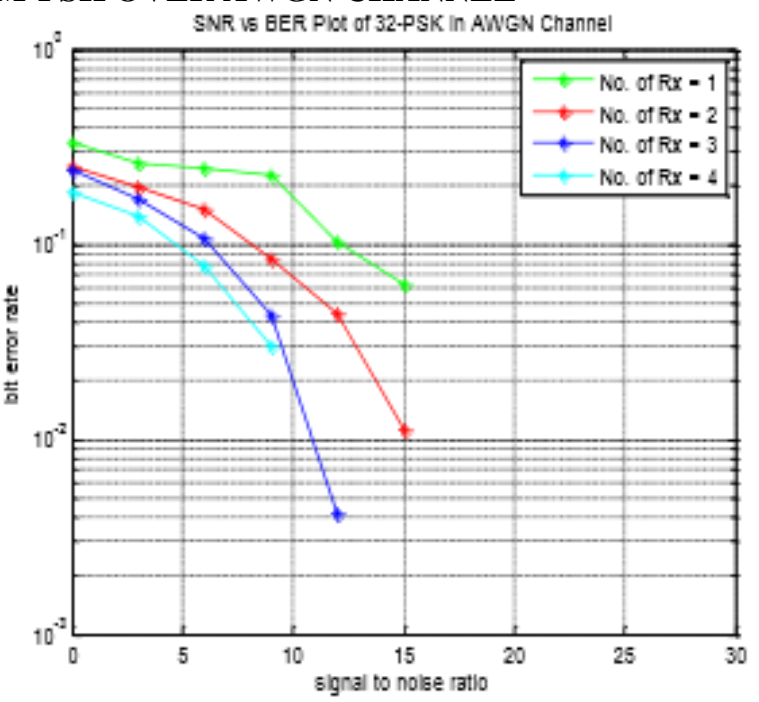

(a) 32-PSK

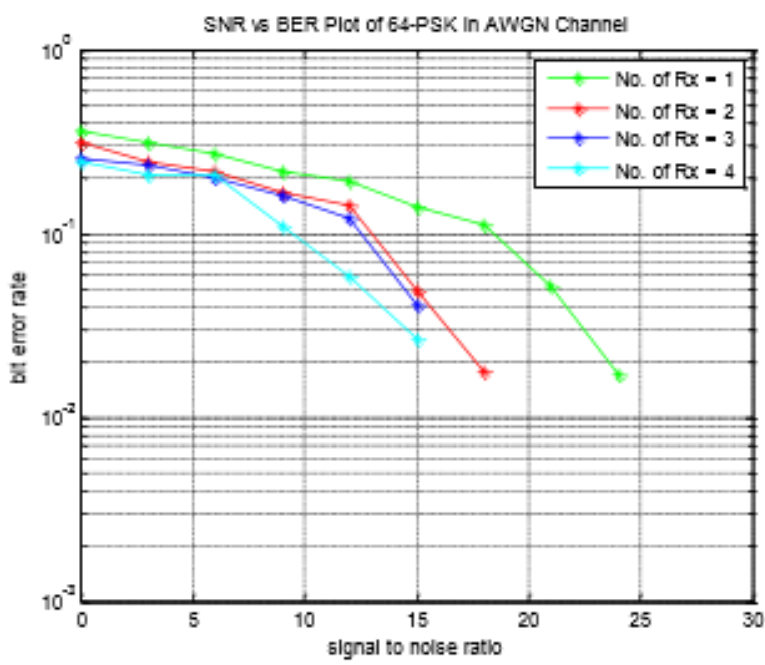

(b) 64-PSK

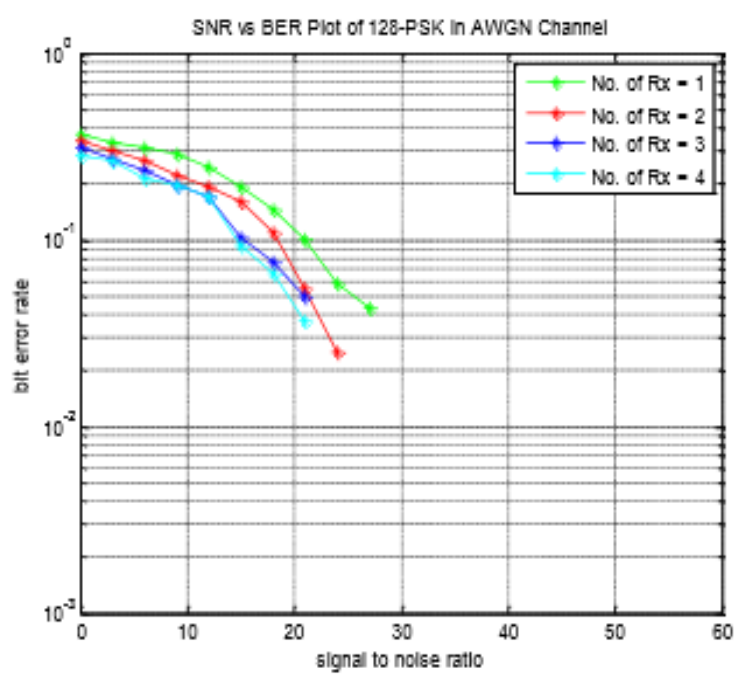

(c) 128-PSK

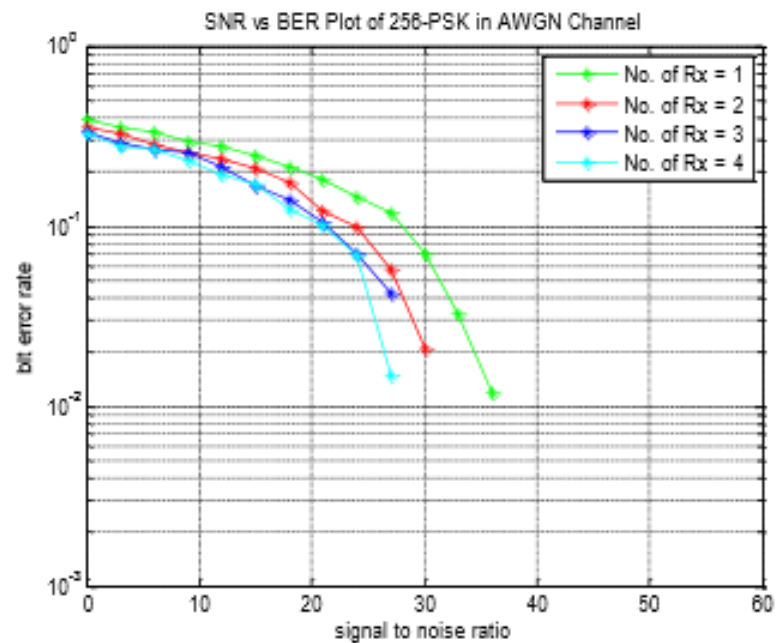

(e) 256-PSK

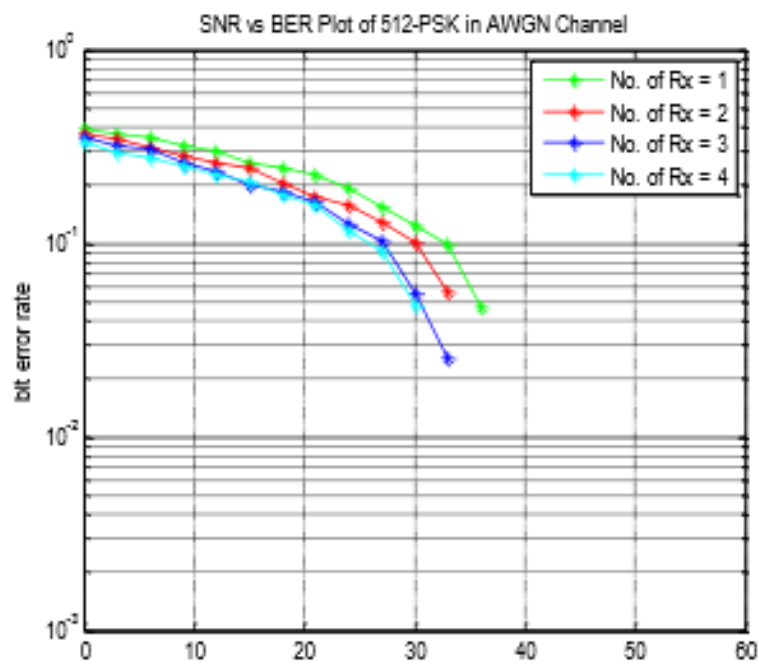

(f) 512-PSK

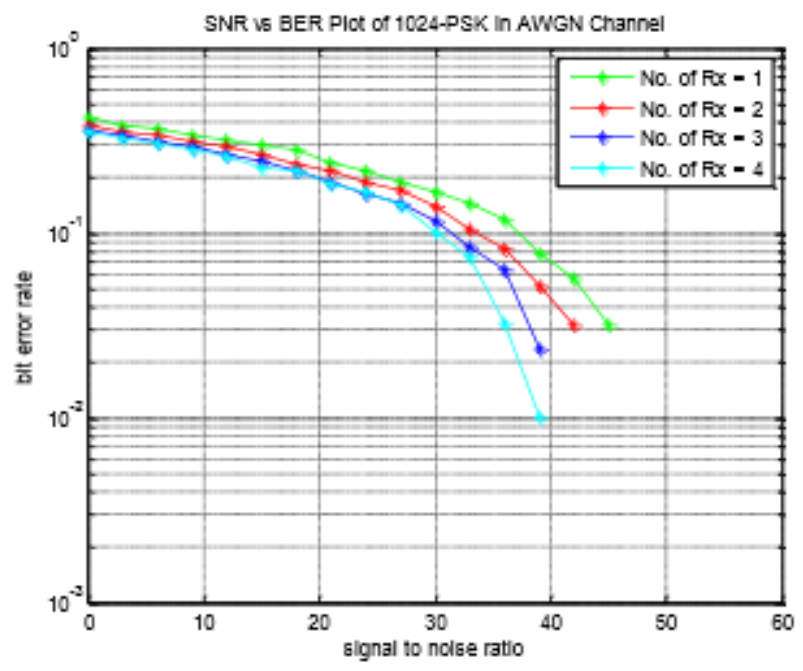

(f) 1024-PSK

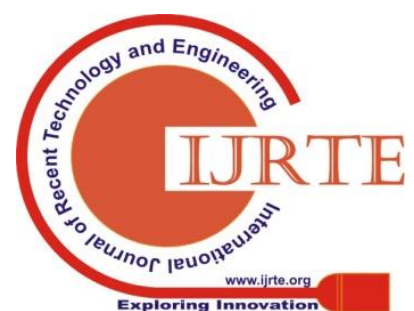




\subsection{M-PSK over Rician channel}

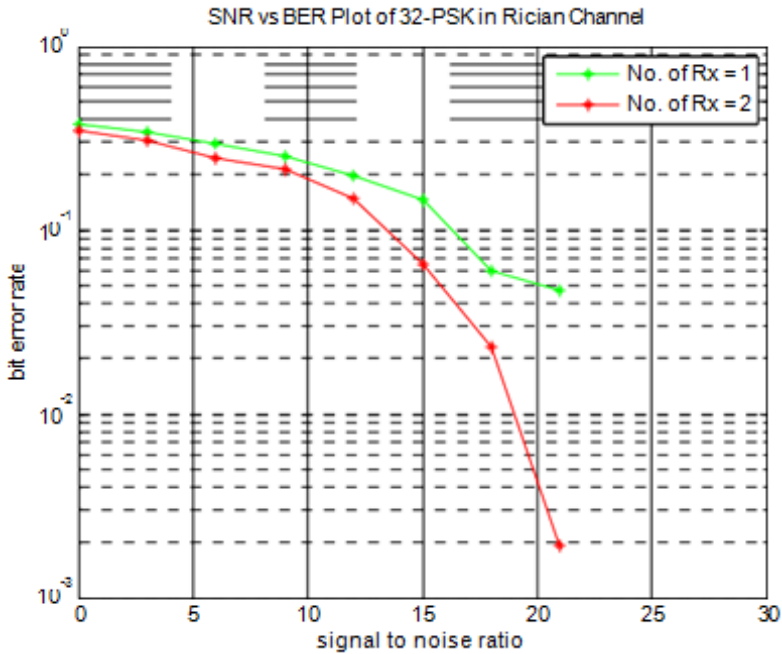

(a) 32-PSK

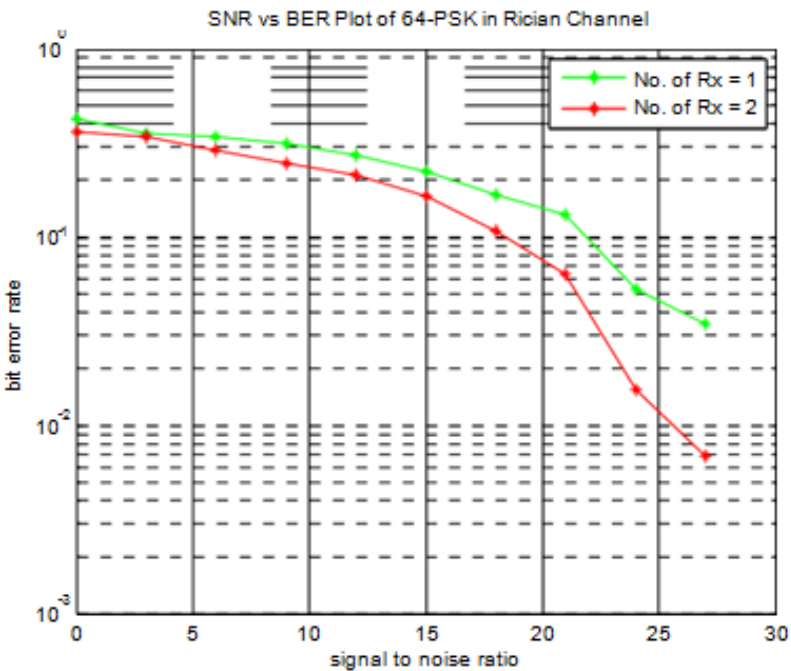

(b) 64-PSK

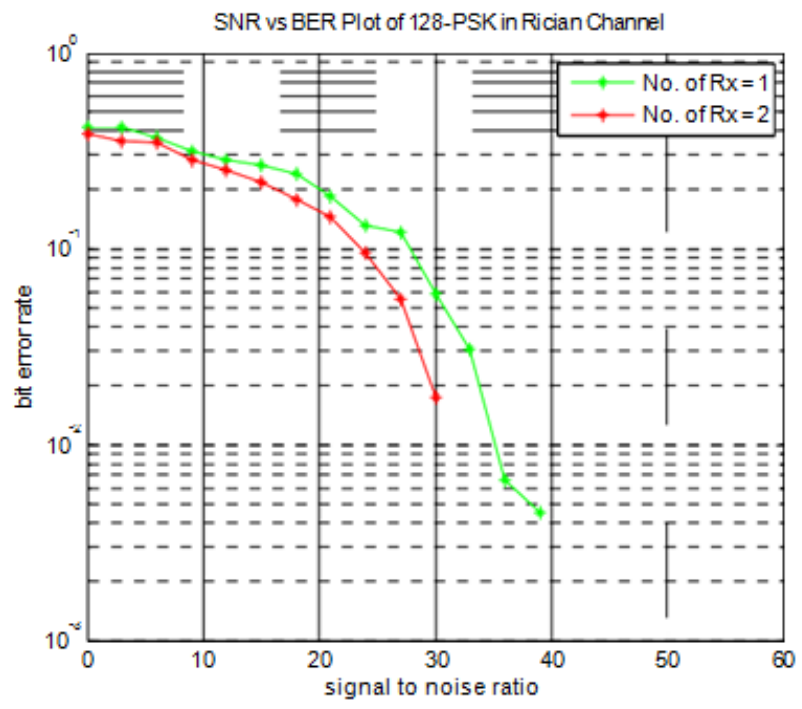

(c) 128-PSK

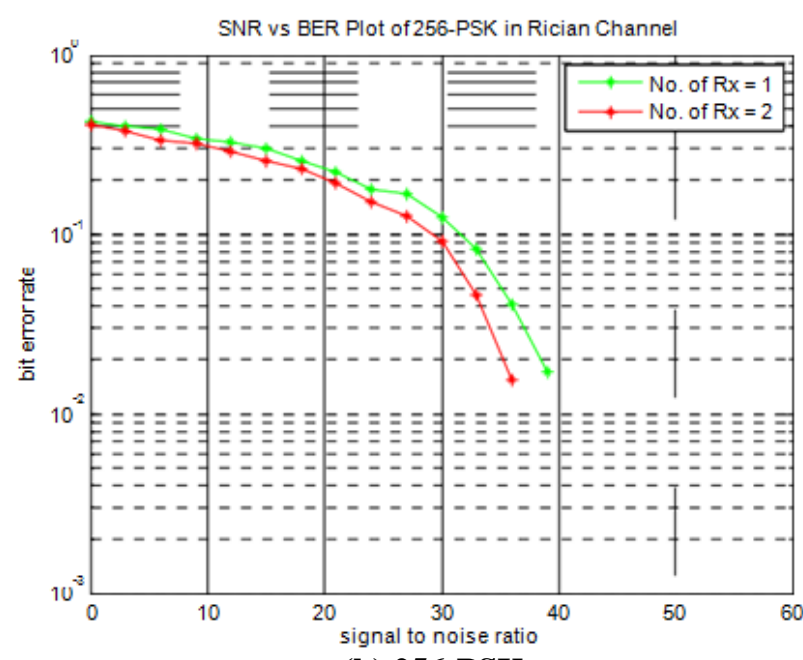

(b) 256-PSK

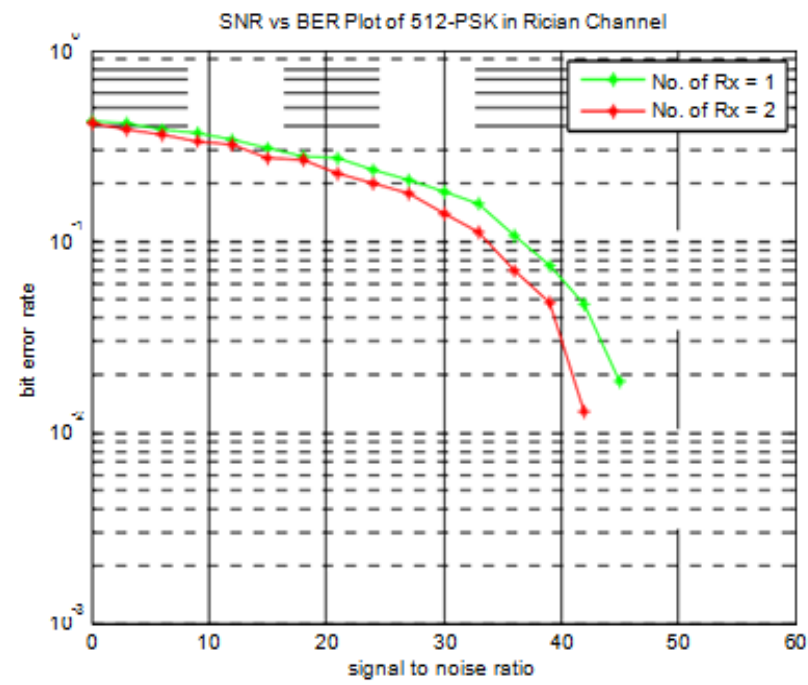

(e)512-PSK

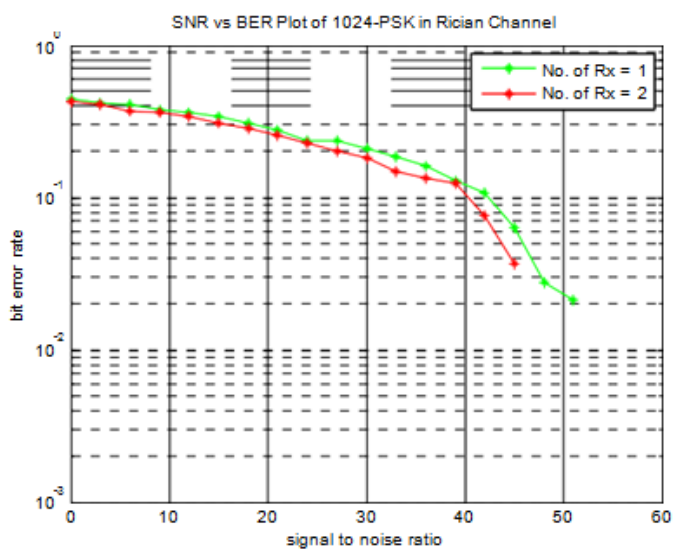

(f) 1024-PSK

The SNR vs BER plots by using M-PSK over Rician channel for MIMO system are shown for different values of ' $M$ ' employing different antenna configurations.

From the above shown results, it can be observed that if the number of receiving antennas in MIMO system increases then the BER value decreases due to space diversity. 
Thus this system provides and operates with better BER performance as compared to the other antenna configurations.

\section{CONCLUSION}

In this paper, the performance analysis of MIMO system over AWGN and Rician fading channels using totally different antenna configurations is bestowed. It may be portrayed from the graphs that the BER keeps on decreasing in MIMO system because of space diversity as increasing the amount of receiving antennas. The receiving antennas used here ranging from $\mathrm{NR}=1$ to $\mathrm{NR}=4$. Spatial diversity techniques are used for the betterment of signal quality and coverage. The multiple receivers or multiple transmitters actually reduce multipath fading and enhance SNR. Thus the proposed system provides better BER performance. But BER is observed to be greater in Rician channel as compared to that of AWGN channel.

\section{REFERENCES}

1. R. W. Heath, "Multimode antenna selection for spatial multiplexing systems with linear receivers", IEEE Transactions on Signal Processing, Vol. 53, Issue 8, pp. 3042-3056, 2005.

2 E. Casas \& C. Leung, "Performance of OFDM/FM scheme for data transmission over fading mobile radio channels", 36th IEEE Vehicular Technology Conference, Vol. 36, Issue 5, pp 103-108, 1986, Dallas, Texas.

3. V. Tarokh, H. Jafarkhani \& A. R. Calderbank, "Space- time block codes from orthogonal designs", IEEE Transactions on Information Theory, Vol. 45, Issue 5, pp. 1456-1467, 1999.

4. G. Ganesan \& P. Stoica. 2001. "Space-time block codes: a maximum SNR approach", IEEE Transactions on Information Theory, Vol. 47, Issue 4, pp. 1650-1656, 2001.

5. P. W. Wolniansky, G. J. Foschini, G. D. Golden \& R. A. Valenzuela, "V-Blast: An architecture for realizing very high data rates over the rich-scattering channel", International Symposium on Signals, Systems and Electronics, pp. 295-300, 1998.

6. X. Zhang, Z. Lv \& W. Wang, "Performance Analysis of Multiuser Diversity in MIMO Systems with Antenna Selection", IEEE Transactions on Wireless Communications, Vol. 7, Issue 1, pp. 15-21, 2008.

7. A. I. Sulyman, "Performance of MIMO Systems With Antenna Selection Over Nonlinear Fading Channels", IEEE Journal of Selected Topics in Signal Processing, Vol. 2, Issue 2, pp. 159-170, 2008.C. Wang, "On the Performance of the MIMO Zero- Forcing Receiver in the Presence of Channel Estimation Error", IEEE Transactions on Wireless Communications, Vol. 6, Issue 3, pp. 805 - 810, 2007.

8. S. G. Kim, D. Yoon, Z. Xu \& S. K. Park, "Performance Analysis of the MIMO Zero-Forcing Receiver over Continuous Flat Fading Channels", IEEE Journal of Selected Areas in Communications, Vol. 20, Issue 7, pp. $324-327,2009$.

9. S. Alamouti, "A simple transmit diversity technique for wireless communications", IEEE Journal on Selected Areas of Communication, Vol. 16, Issue 8, pp. 1451-1458, 1998.

10. V. Tarokh, N. Seshadri \& A. R. Calderbank, "Space- Time Codes for High Data Rate Wireless Communication: Performance Criterion and Code Construction", IEEE Transactions on Information Theory, Vol. 44, Issue 2, pp. 744-765, 1998.

11. C. Chen, "Performance Analysis of Scheduling in Multiuser MIMO Systems with Zero-Forcing Receivers", IEEE Journal of Selected Areas in Communications, Vol. 25, Issue 7, pp. 1435-1445, 2007.

12 N. S. Kumar, G. J. Foschini, G. D. Golden \& R. A. Valenzuela, "Bit Error Rate Performance Analysis of ZF, ML and MMSE Equalizers for MIMO Wireless Communication Receiver", European Journal of Scientific Research, Vol. 59, Issue 4, pp. 522-532, 2011. 